\title{
Loss of opioid binding protein/cell adhesion molecule-like gene expression in gastric cancer
}

\author{
NING ZHANG ${ }^{1}$, JIDE XU $^{2}$, YUHONG WANG $^{3}$, XUHUA HENG $^{4}$, LITENG YANG $^{1}$ and XIANGBIN XING ${ }^{5}$ \\ ${ }^{1}$ Department of Respiratory Disease, Affiliated LuoHu Hospital of Shenzhen University, Shenzhen, \\ Guangdong 518001; ${ }^{2}$ Department of Physiology, Guangzhou Medical University; ${ }^{3}$ Department of Endoscopy, \\ Sun Yat-sen University Cancer Center, Guangzhou, Guangdong 510000; ${ }^{4}$ Department of Cardiogy, \\ Affiliated Hospital of Panzhihua University, Panzhihua, Sichuan 617000; ${ }^{5}$ Department of Gastroenterology, \\ The First Affiliated Hospital of Sun Yat-sen University, Guangzhou, Guangdong 510000, P.R. China
}

Received October 16, 2016; Accepted May 17, 2017

DOI: $10.3892 / \mathrm{ol} .2018 .8562$

\begin{abstract}
Previous studies have reported that the expression of the opioid binding protein/cell adhesion molecule-like (OPCML) gene was frequently downregulated in various of types of cancer. However, little is known regarding the expression of the OPCML gene in gastric cancer. The present study identified that OPCML was downregulated in the gastric cancer SGC7901, KATO III, MKN45, MKN74, SNU1, AGS, N87 and a gastric mucosa cell line GES1, compared with normal gastric tissues by reverse transcription-quantitative polymerase chain reaction (RT-qPCR). To investigate whether the downregulation of OPCML was due to promoter hypermethylation, the methylation of the OPCML promoter was assessed by methylation-specific polymerase chain reaction. Hypermethylation of the OPCML promoter was observed in the gastric cancer MKN45 cell lines, but was not as evident in normal gastric tissue. The methylation inhibitor 5-aza-2'-deoxycytidine was used to remove the methylation of the OPCML gene promoter, following which the expression of OPCML was restored. In addition, the function of the OPCML gene was studied in vitro, and it was found that the restoration expression of OPCML could lead to the suppression of cell growth. In conclusion, the present study has shown that OPCML, which acts as a tumor suppressor, was silenced in
\end{abstract}

Correspondence to: Professor Xiangbin Xing, Department of Gastroenterology, The First Affiliated Hospital of Sun Yat-sen University, 58 Zhongshan II Road, Guangzhou, Guangdong 510000, P.R. China

E-mail: 14701146@qq.com

Professor Liteng Yang, Department of Respiratory Disease, Affiliated LuoHu Hospital of Shenzhen University, 47 Youyi Road, Shenzhen, Guangdong 518001, P.R. China

E-mail: yang20002004@163.com

Key words: opioid binding protein/cell adhesion molecule-like gene expression, gastric cancer, reverse transcription-polymerase chain reaction, methylation specific polymerase chain reaction gastric cancer cell lines via aberrant hypermethylation of the promoter $\mathrm{CpG}$ islands, which may provide a novel molecular approach for the early diagnosis of gastric cancer.

\section{Introduction}

Gastric cancer is the second most common cause of cancer-related mortality worldwide (1). In recent studies, surgical resection along with chemo-radiation demonstrated significant improvement compared with surgery alone; however, numerous patients with gastric cancer have advanced or metastatic diseases at diagnosis (2-5). The molecular mechanisms involved in tumor development and progression remain unclear in gastric cancer (6). A number of studies have reported that $\mathrm{CpG}$ island methylation leads to inactivation and silencing of respective tumor suppressor genes, including COX-2, APC, HPP1 and DAPK in gastric cancer $(7,8)$. To improve the prognosis of gastric cancer patients, a greater understanding of the biological mechanisms of gastric cancer progression and novel therapeutic methods is required.

Opioid binding protein/cell adhesion molecule-like (OPCML), located on 11q25, is a glycosylphosphatidylinositol (GPI)-anchored cell adhesion-like molecule; it is strongly associated with cell growth, invasion, and metastasis and tumorigenesis (9). OPCML is widely expressed in adult tissues; however, in cancer tissues of various types, including nasopharyngeal carcinoma, hepatocellular carcinoma, bladder cancer, ovarian cancer and cervical carcinoma, its promoter is often methylated and its expression decreased (9-12). To the best of our knowledge, little is known regarding the association between OPCML and the occurrence and development of gastric cancer. Therefore, the aim of the present study was to investigate the mRNA expression of OPCML and the degree of $\mathrm{CpG}$ island methylation in human gastric cancer cell lines, and to elucidate the molecular mechanisms that may underlie the loss of OPCML expression in gastric cancer cell lines.

\section{Materials and methods}

Cell lines and tissue. The human gastric cancer SGC7901, MKN74, MKN45, KATO III, SNU1 cell lines were obtained 
from RIKEN BioResource Center (Tsukuba, Japan) and the cell lines AGS, N87 and the immortal gastric mucosal GES1 cell lines were obtained from the American Type Culture Collection (Manassas, VA, USA). All cell lines were maintained in RPMI-1640 medium (HyClone; GE Healthcare Life Sciences, Logan, UT, USA) with $10 \%$ fetal bovine serum in a $5 \% \mathrm{CO}_{2}$ atmosphere at $37^{\circ} \mathrm{C}$. In addition, normal gastric tissue samples were obtained from the gastric antrum (female, 59 years) and corpus gastricum (female, 43 years) by biopsy at the Affiliated LuoHu Hospital of Shenzhen University in February 2013. The present study was approved by the Ethics Committee of the Affiliated LuoHu Hospital of Shenzhen University; written informed consent was obtained from patients.

Reverse transcription-polymerase chain reaction ( $R T-P C R)$. The total RNA of all aforementioned cell lines and normal gastric tissue samples were extracted using the RNA-lyase Mini kit (Macherey-Nagel GmbH and Co., Düren, Germany), according to the manufacturer's protocol. For each RNA sample, $1 \mu \mathrm{g}$ was reverse-transcribed using the RevertAid First Strand cDNA Synthesis kit (Fermentas; Thermo Fisher Scientific, Inc., Waltham, MA, USA). The cDNA was then used to amplify the desired gene with specific primers using PCR Amplification Reaction kit (Promega Corporation, Madison, WI, USA). The number of PCR cycles was suitable to each gene for complete linear amplification. The PCR thermocycling conditions were as follows: Initial denaturation at $95^{\circ} \mathrm{C}$ for $5 \mathrm{~min}, 35$ cycles including denaturation at $95^{\circ} \mathrm{C}$ for $30 \mathrm{sec}$, annealing at $60^{\circ} \mathrm{C}$ for $30 \mathrm{sec}$, elongation at $7^{\circ} \mathrm{C}$ for $30 \mathrm{sec}$, and then $72^{\circ} \mathrm{C}$ for $10 \mathrm{~min}$. PCR primers were designed to amplify a 126-bp cDNA fragment of the human OPCML gene (forward, 5'-ACACCACTGGGTGGAGAAAG-3' and reverse, 5'-AAGGGCAGCTTGCAGTACAT-3'). The mitochondrial ribosomal protein $\mathrm{S} 12$ was used as normalization reference (forward, 5'-GCATTGCTGCTGGAGGTGTAAT-3' and reverse, 5'-CTGCAACCAACCACTTTACGG-3'). The size of the mitochondrial ribosomal protein S12 gene was 306-bp. To evaluate the PCR products, the products were electrophoresed on 1\% agarose gel in Tris base-boric acid-EDTA buffer solution (Sigma-Aldrich; Merck KGaA; Darmstadt, Germany). To analyze the electrophoresis results, the samples were stained with $1 \mathrm{mg} / \mathrm{ml}$ ethidium bromide solution for $1 \mathrm{~min}$ at room temperature, and visualized by an UV transilluminator apparatus.

Sodium bisulfite genomic sequencing. Genomic DNA from the gastric cancer cell line MKN45 was extracted using the High Pure PCR Template Preparation kit (Machery-Nagel GmbH), which were selected at random from the gastric cell lines. A total of $1 \mu \mathrm{g}$ DNA was then treated using the $\mathrm{CpGenome}^{\mathrm{TM}}$ DNA Modification kit (Merck KGaA, Darmstadt, Germany) according to the manufacturer's protocol. Methylated primer sequences were as follows: Forward, 5'-CGTTTAGTTTTT CGTGCGTTC-3' and reverse, 5'-CGAAAACGCGCAACC GACG-3'. The size of the purpose fragment gene is 129-bp. The unmethylated primer sequences were as follows: Forward, 5'-TTTGTTTAGTTTTTTGTGTGTTTG-3' and reverse, 5'-CAAAACAAAAACACACAACAACA-3'. The size of the purpose fragment gene was 136-bp. The PCR thermocycling conditions were as follows: $98^{\circ} \mathrm{C}$ for $10 \mathrm{~min}$ followed by 40 cycles with denaturation at $97^{\circ} \mathrm{C}$ for $50 \mathrm{sec}$, annealing at $60^{\circ} \mathrm{C}$ for $30 \mathrm{sec}$, elongation at $72^{\circ} \mathrm{C}$ for $40 \mathrm{sec}$, and a final extension step of $72^{\circ} \mathrm{C}$ for 10 min using PCR Amplification Reaction kit (Promega Corporation). The methylated specific primer used to amplify the gene that the promoter was methylated. Meanwhile the unmethylated specific primer used to amplify the unmethylated DNA, following methylated and unmethylated DNA being completely bisulfite modified.

Treatment of cells with 5-aza-2'-deoxycytidine (5-AZA). The gastric cancer AGS cell line and the immortal gastric mucosal GES1 cell line were selected at random and seeded at a density of $1 \times 10^{6}$ cells on a $60-\mathrm{mm}$ dish. Following a $24 \mathrm{~h}$ incubation period in a $5 \% \mathrm{CO}_{2}$ atmosphere at $37^{\circ} \mathrm{C}$, cells were treated with $10 \mu \mathrm{mol} / 1$ of 5-AZA (Sigma-Aldrich; Merck KGaA). The same concentration of DMSO was also used as a control for nonspecific solvent treatment with these cells. The cells were extracted $72 \mathrm{~h}$ after treatment with 5-AZA using the RNA-lyase Mini kit (Macherey-Nagel GmbH and Co.), and $1 \mu \mathrm{g}$ RNA was reverse-transcribed using the RevertAid First Strand cDNA Synthesis kit, and then the cDNA was used to amplify OPCML gene using PCR Amplification Reaction kit, as previously described by $\mathrm{Gu}$ et al (7).

Cells transfected with pcDNA3.1+/OPCML detected by $R T$-PCR. The gastric cancer cell lines MKN45, AGS, and 293 were transfected with pcDNA3.1+/OPCML or pcDNA 3.1 vector alone (Beijing Fungenome Company, Beijing, China), using the transfection reagent Lipofectamine ${ }^{\circledR} 2000$ (Invitrogen; Thermo Fisher Scientific, Inc.). At $48 \mathrm{~h}$ post-transfection, the total RNA of cells was extracted using the RNA lyase Mini kit (Machery-Nagel $\mathrm{GmbH}$ ), according to the manufacturer's protocol. For each RNA sample, $1 \mu \mathrm{g}$ was reverse-transcribed using a First Strand cDNA Synthesis kit (Fermentas; Thermo Fisher Scientific, Inc.) according to the manufacturer's protocol. The PCR primers were designed to amplify a 828-bp cDNA fragment of the human OPCML gene (forward, 5'-TCCCCAAAGCTATGGACAAC-3' and reverse, 5'-GCCCATACAATGTGATG-3'). The conditions on the polymerase chain reaction was set as follows: First denaturation at $94^{\circ} \mathrm{C}$ for $5 \mathrm{~min}$, followed by 35 cycles with denaturation at $94^{\circ} \mathrm{C}$ for $30 \mathrm{sec}$, annealing at $60^{\circ} \mathrm{C}$ for $30 \mathrm{sec}$, elongation at $72^{\circ} \mathrm{C}$ for $30 \mathrm{sec}$, and a final elongation step of $72^{\circ} \mathrm{C}$ for $10 \mathrm{~min}$ using PCR Amplification Reaction kit (Promega Corporation). The mitochondrial ribosomal protein S12 was used as normalization reference gene (forward 5'-GCATTGCTGCTGGAG GTGTAAT-3' and reverse, 5'-CTGCAACCAACCACTTTA CGG-3'). The size of the mitochondrial ribosomal protein $\mathrm{S} 12$ gene was 306-bp. To evaluate the PCR products, the products were electrophoresed on $1 \%$ agarose gel in Tris base-boric acid-EDTA buffer solution (Sigma-Aldrich; Merck KGaA). To analyze the electrophoresis results, the samples were stained with $1 \mathrm{mg} / \mathrm{ml}$ of ethidium bromide solution for $1 \mathrm{~min}$ at room temperature, and visualized by an UV transilluminator apparatus as aforementioned.

Colony formation assay. Gastric cancer cell lines AGS were transfected with pcDNA3.1+/OPCML and pcDNA 3.1 vector, as aforementioned. After $48 \mathrm{~h}$ of transfection, cells were 
seeded $\left(1 \times 10^{4}\right)$ on a $60 \mathrm{~mm}$ dish, and selected for 2 weeks in the presence of $400 \mu \mathrm{g} / \mathrm{ml} \mathrm{G} 418$ (Invitrogen; thermo Fisher Scientific, Inc.). Surviving colonies ( $\geq 50$ cells per colony) were counted following staining with $5 \%$ crystal violet solution for $10 \mathrm{~min}$ at room temperature. The data were obtained from three independent cell cultures and experiments were repeated three times.

Statistical analysis. Results are presented as the mean \pm standard deviation and were analyzed using SPSS 16.0 (SPSS, Inc., Chicago, IL, USA) and plotted using Graphpad Prism 6.0 (GraphPad Software, Inc., La Jolla, CA, USA). Data with two groups were compared using Student's unpaired t-test. $\mathrm{P}<0.05$ was considered to indicate a statistically significant difference.

\section{Results}

OPCML expression in gastric cancer cell lines and normal gastric tissue. In the present study, OPCML expression was assessed using RT-PCR in the gastric cancer AGS, SGC7901, KATO III, MKN74, N87, SNU and MKN45 cell lines, in the immortal gastric mucosa GES1 cell line and in normal gastric tissue. As Fig. 1 indicates, the expression of the OPCML gene was reduced in AGS, SGC7901, KATO III, MKN74, N87, SNU, MKN45 and GES1 cell lines, compared with normal gastric tissue (Fig. 1).

OPCML downregulation was mediated by promoter methylation in gastric cancer cell lines. To investigate the role of putative OPCML gene losses in gastric cells, methylation-specific PCR (MSP) was performed in MKN45 cells to evaluate the methylation of promoter $\mathrm{CpG}$ islands using specific MSP primers. The data revealed that hypermethylation of the OPCML promoter commonly occurred in MKN45 cells, and there was no methylation in normal gastric tissues (Fig. 2). To verify whether $\mathrm{CpG}$ island methylation directly mediates OPCML silencing, the gastric cancer cell line MKN45 and immortal gastric mucosa cell GES1 were treated with the demethylating agent 5-AZA. Following this treatment, OPCML expression was observed to be markedly restored following drug treatment in MKN45 cells (Fig. 3).

OPCML suppressed gastric cancer colony formation. To investigate OPCML gene function further, the gastric cancer cell MKN45, AGS lines and 293 cells were transfected with the pcDNA3.1+/OPCML and pcDNA 3.1 vector (Fig. 4). Fewer cells transfected with the OPCML gene adhered to the culture dish compared with those transfected with an empty vector $(\mathrm{P}<0.05$; Figs. 5 and 6$)$. These results indicated that the OPCML gene possesses the ability to inhibit colony formation in gastric cancer.

\section{Discussion}

OPCML belongs to the IgLON family of immunoglobulin domain-containing glycosylphosphatidylinositol-anchored cell adhesion molecules, which includes opioid-binding cell adhesion molecule, neurotbrimin, neuronal growth regulator 1 and limbic system-associated membrane protein. It has been

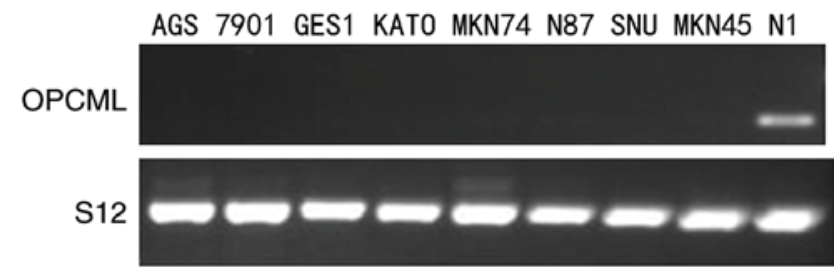

Figure 1. Identification of OPCML expression in cell lines AGS, SGC7901, GES1, KATO, MKN74, SNU, MKN45 and N1 by reverse transcription-polymerase chain reaction analysis. OPCML, Opioid binding protein/cell adhesion molecule-like; N1, normal gastric tissue; S12, mitochondrial ribosomal protein S12.
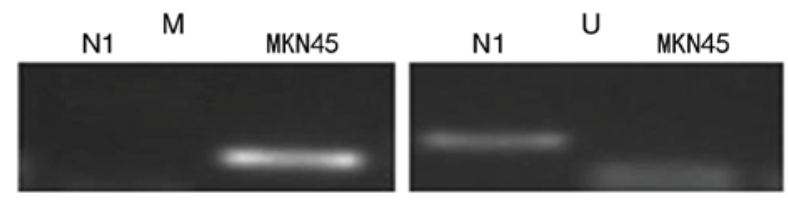

Figure 2. Frequent methylation of the OPCML promoter of $\mathrm{CpG}$ islands in MKN45 analyzed by a methylation specific polymerase chain reaction. OPCML, Opioid binding protein/cell adhesion molecule-like; M, methylated; U, unmethylated; N1, normal gastric tissue.

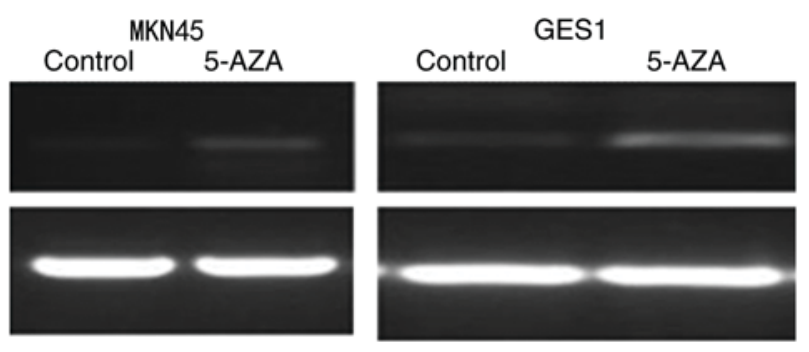

Figure 3. Changes to OPCML expression levels prior to and following incubation with 5-AZA treatment. OPCML, Opioid binding protein/cell adhesion molecule-like; S12, mitochondrial ribosomal protein S12; 5-AZA, 5-aza-2'-deoxycytidine.

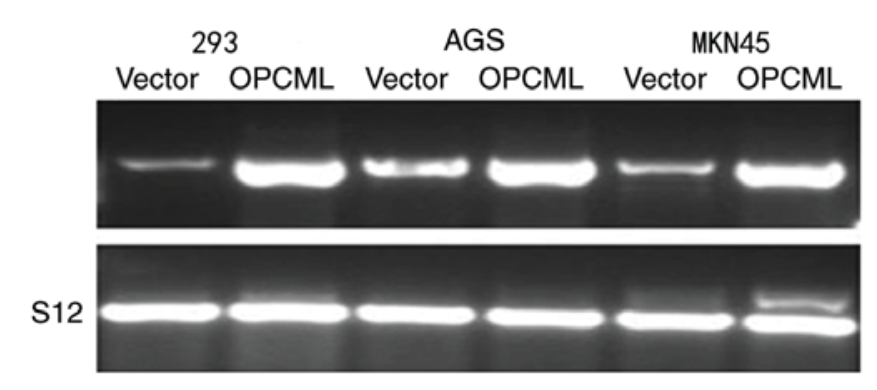

Figure 4. Transfection of gastric cancer cell lines MKN45, AGS and cell HEK293 with pcDNA3.1+/OPCML or pcDNA 3.1 vectors was assessed by reverse transcription-polymerase chain reaction. OPCML, Opioid binding protein/cell adhesion molecule-like.

reported that IgLONs serve a notable role in cell-cell recognition and adhesion (13-18). OPCML, which acts as a cell adhesion molecule, contains several protein-protein interaction domains, including the three C2-like Ig domains, which are commonly found in cell surface adhesion molecules and receptor proteins. Through these domains, OPCML was demonstrated to modulate functions of growth promotion or inhibition in tumor cells. 


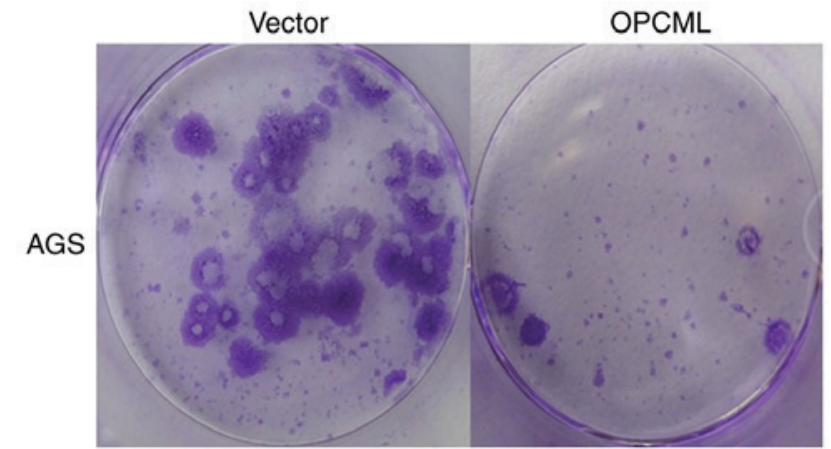

Figure 5. Effects of OPCML on colony formation in AGS cells. OPCML, Opioid binding protein/cell adhesion molecule-like.

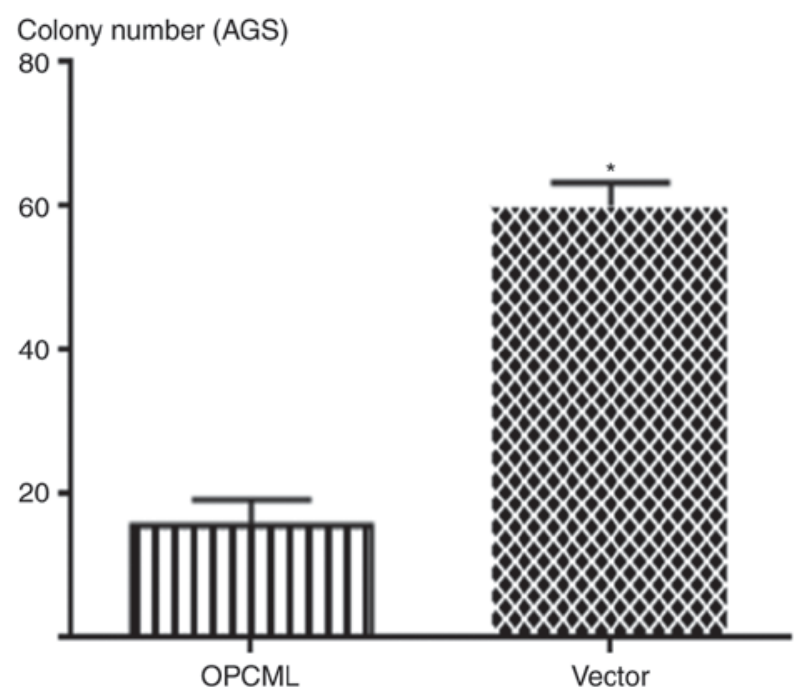

Figure 6. Histogram concerning the effects of OPCML gene on the colony formation in AGS cells. "P<0.05. OPCML, Opioid binding protein/cell adhesion molecule-like; vector, empty vector.

OPCML was the first member of the IgLON family identified to possess tumor suppressor functions in multiple cancer types, which are frequently epigenetically and genetically silenced at the early stage of carcinogenesis. The loss of OPCML may reduce heterodimeric complex formation and cell-cell adhesion, therefore damaging the corresponding signaling pathways and promoting the progress of carcinogenesis (13-18).

In the present study, the expression of OPCML in the SGC7901, KATO III, MKN45, MKN74, SNU1, AGS and N87 cell lines the immortal gastric mucosal GES1 cell line and normal gastric tissue were assessed by RT-PCR analysis. OPCML was demonstrated to be downregulated in SGC7901, KATO III, MKN45, MKN74, SNU1, AGS, N87 and GES1 cell lines, when compared with normal gastric tissue. This observation was corroborated by Wang et al (6) who also reported that the expression of OPCML was downregulated in patients with gastric cancer compared with normal gastric tissue by RT-PCR analysis.

OPCML acts as a tumor suppressor in multiple cancer types, including nasopharyngeal carcinoma, bladder cancer, ovarian cancer, cervical carcinoma, esophageal carcinoma and hepatocellular carcinoma; recent studies have reported that the loss or downregulation of OPCML expression is associated with OPCML gene promoter methylation (18-20). However, to the best of our knowledge, little is known regarding the association between OPCML expression and promoter methylation in gastric cancer. Therefore, in the present study, to confirm whether OPCML gene promoter methylation is the cause of attenuated OPCML expression, MSP analysis was performed in the MKN45 cell line using the CpGenome ${ }^{\mathrm{TM}}$ DNA Modification kit and hypermethylation of the OPCML promoter was demonstrated to occur in MKN45.

In the present study the gastric cancer cell line MKN45 and the immortal gastric mucosal cell line GES1 were treated with the methylation inhibitor 5-AZA and it was demonstrated that treatment with 5-AZA was able to restore or upregulate the expression of OPCML mRNA in these cells. To investigate OPCML gene function, the gastric cancer cell line AGS was transfected with the pcDNA3.1+/OPCML and pcDNA 3.1 vector. Ectopic expression of OPCML in gastric cell lines with endogenous silencing resulted in the inhibition of cell colony formation, indicating that OPCML acts as a broad tumor suppressor.

DNA methylation is an epigenetic phenomenon that affects gene expression without altering the DNA sequence (20-24). Aberrant supermethylation occurs in promoter $\mathrm{CpG}$ islands, and is a mechanism by which tumor suppressor genes are silenced and, in certain circumstance, may be an important mechanism (20-24). The present study has demonstrated that OPCML, which acts as a broad tumor suppressor gene, is silenced in gastric cancer cell lines via the aberrant supermethylation of promoter $\mathrm{CpG}$ islands. This typically occurred prior to the development of clinical manifestations in patients and the obtaining of radiographic evidence, and therefore may provide a novel molecular approach for the early diagnosis of gastric cancer.

In the present study OPCML gene function in vivo was not investigated and the OPCML protein expression in gastric cancer cell lines and normal gastric tissue is unknown. Future functional studies are required to clarify its role in signaling pathways, which in turn may result in the identification of further molecular targets in gastric cancer.

\section{Acknowledgements}

Not applicable.

\section{Funding}

This study was supported by the National Natural Science Foundation of China (grant no. 81000887).

\section{Availability of data and materials}

The datasets used during the current study are available from the corresponding author on reasonable request.

\section{Authors' contributions}

NZ and XX were responsible for drafting the manuscript. NZ, YW and XH contributed the experiments. XX, YW, JX and $\mathrm{XH}$ contributed to analysis and interpretation of data. LY and XX contributed to conducting the study. All authors read and approved the final manuscript. 


\section{Ethics approval and consent to participate}

The present study was approved by the Ethics Committee of the Affiliated LuoHu Hospital of Shenzhen University and written informed consent was obtained from all patients.

\section{Consent for publication}

The patients provided written informed consent for the publication of any associated data.

\section{Competing interests}

The authors declare no competing interests.

\section{References}

1. Kim SJ, Wang YG, Lee HW, Kang HG, La SH, Choi IJ, Irimura T, Ro JY, Bresalier RS and Chun KH: Up-regulation of neogenin-1 increases cell proliferation and motility in gastric cancer. Oncotarget 5: 3386-3398, 2014.

2. Wu HH,Lin WC and Tsai KW: Advances in molecular biomarkers for gastric cancer: miRNAs as emerging novel cancer markers. Expert Rev Mol Med 16: e1, 2014.

3. Zhang Y and Wu S: Novel therapy for advanced gastric cancer. World J Gastrointest Oncol 7: 263-270, 2015

4. Yan S, He F, Luo R, Wu H, Huang M, Huang C, Li Y and Zhou Z: Decreased expression of BRCA1-associated protein 1 predicts unfavorable survival in gastric adenocarcinoma. Tumor Biol 37: 6125-6133, 2016

5. Wen R, Gao F, Zhou CJ and Jia YB: Polymorphisms in mucin genes in the development of gastric cancer. Word J Gastrointest Oncol 7: 328-337, 2015

6. Wang L, Zhu JS, Song MQ, Chen GQ and Chen JL: Comparison of gene expression profiles between primary tumor and metastatic lesions in gastric cancer patients using laser microdissection and cDNA microarray. Word J Gastroenterol 12: 6949-6954, 2006.

7. Gu P, Xing X, Tänzer M, Röcken C, Weichert W, Ivanauskas A, Pross M, Peitz U, Malfertheiner P, Schmid RM and Ebert MP: Frequent loss of TIMP-3 expression in progression of esophageal and gastric adenocarcinomas. Neoplasia 10: 563-572, 2008.

8. Kobayashi K, Inokuchi M, Takagi Y, Otsuki S, Fujimori Y, Sato Y, Yanaka Y, Higuchi K, Aburatani T, Tomii C, et al: Prognostic significance of PAK4 expression in gastric cancer J Clin Pathol 69: 580-585, 2016.

9. Li C, Tang L, Zhao L, Li L, Xiao Q, Luo X, Peng W, Ren G, Tao Q and Xiang T: OPCML is frequently methylated in human colorectal cancer and its restored expression reverses EMT via downregulation of smad signaling. Am J Cancer Res 5: $1635-1648,2015$

10. Sanz R, Ferraro GB and Fournier AE: IgLON cell adhesion molecules are shed from the cell surface of cortical neurons to promote neuronal growth. J Biol Chem 290: 4330-4342, 2015.
11. Minhas HM, Pescosolido MF, Schwede M, Piasecka J, Gaitanis J, Tantravahi U and Morrow EM: An unbalanced translocation involving loss of 10q26.2 and gain of 11q25 in a pedigree with autism spectrum disorder and cerebellar juvenile pilocytic astrocytoma. Am J Med Genet A 161A: 787-791, 2013.

12. Zhou F, Tao G, Chen X, Xie W, Liu M and Cao X: Methylation of OPCML promoter in ovarian cancer tissues predicts poor patient survival. Clin Chem Lab Med 52: 735-742, 2014.

13. Zhou F, Ma M, Tao G, Chen X, Xie W, Wang Y and Cao X: Detection of circulating methylated opioid binding protein/cell adhesion molecule-like gene as a biomarker for ovarian carcinoma. Clin Lab 60: 759-765, 2014.

14. Wu SY and Sood AK: New roles opined for OPCML. Cancer Discov 2: 115-116, 2012

15. McKie AB, Vaughan S, Zanini E, Okon IS, Louis L, de Sousa C, Greene MI, Wang Q, Agarwal R, Shaposhnikov D, et al: The OPCML tumor suppressor functions as a cell surface repressor-adaptor, negatively regulating receptor tyrosine kinases in epithelial ovarian cancer. Cancer Discov 2: 156-171, 2012.

16. Amoenpisutt $R$, Proungvitaya $S$, Jearanaikoon $P$ and Limpaiboon T: DNA methylation level of OPCML and SFRP1: A potential diagnostic biomarker of cholangiocarcinoma. Tumor Biol 36: 4973-4978, 2015

17. Rein BJ, Gupta S, Dada R, Safi J, Michener C and Agarwal A: Potential markers for detection and monitoring of ovarian cancer. J Oncol 2011: 475983, 2011.

18. Chen W, Xiang J, Chen DF, Ni BB, Chen H, Fan XJ, Wang PN, Song SX, Fang LK, Xiao HY, et al: Screening for differentially methylated genes among human colorectal cancer tissues and normal mucosa by microarray chip. Mol Biol Rep 40: 3457-3464, 2013.

19. Reed JE, Dunn JR, du Plessis DG, Shaw EJ, Reeves P, Gee AL, Warnke PC, Sellar GC, Moss DJ and Walker C: Expression of cellular adhesion molecule 'OPCML' is down-regulated in gliomas and other brain tumours. Neuropathol Appl Neurobiol 33: 77-85, 2007.

20. Amornpisutt R, Sriraksa R and Limpaiboon T: Validation of methylation-sensitive high resolution melting for the detection of DNA methylation in cholangiocarcinoma. Clin Biochem 45: 1092-1094, 2012.

21. Cui Y, Ying Y, van Hasselt A, Ng KM, Yu J, Zhang Q, Jin J, Liu D, Rhim JS, Rha SY, et al: OPCML is a broad tumor suppressor for multiple carcinomas and lymphomas with frequently epigenetic inactivation. PLoS One 3: e2990, 2008.

22. Wang B, Yu L, Yang GZ, Luo X and Huang L: Applicaion of multiplex nested methylated specific PCR in early diagnosis of epithelial ovarian cancer. Asian Pac J Cancer Prev 16: 3003-3007, 2015.

23. Ye F, Zhang SF, Xie X and Lu WG: OPCML gene promoter methylation and gene expression in tumor and stroma cells of invasive cervical carcinoma. Cancer Invest 26: 569-574, 2008.

24. Zhang Q, Hu G, Yang Q, Dong R, Xie X, Ma D, Shen K and Kong B: A multiplex methylation-specific PCR assay for the detection of early-stage ovarian cancer using cell-free serum DNA. Gynecol Oncol 130: 132-139, 2013. 